\title{
Diagnóstico de enfermagem comunicação verbal prejudicada na prática clínica: uma revisão integrativa
}

\author{
Nursing diagnosis impaired verbal communication in clinical practice: an integrative \\ review
}

\section{Diagnóstico de enfermería comunicación verbal perjudicada en la práctica clínica: una revisión integradora}

\section{Recebido: 28/11/2015 \\ Aprovado: 05/04/2016 \\ Publicado: 01/05/2016}

Ana Cláudia Giesbrecht Puggina ${ }^{1}$ Monica Martins Trovo ${ }^{2}$ Chaiane Amorim Biondo ${ }^{3}$ Ingrid de Almeida Barbosa ${ }^{4}$ Mariana Santos 5 Maria Júlia Paes da Silva6

Este estudo tem como objetivo identificar na literatura o uso na prática clínica do diagnóstico de enfermagem Comunicação Verbal Prejudicada em adultos e idosos. Trata-se de uma revisão integrativa da literatura nas bases de dados LILACS, BDENF, Coleciona SUS, CINAHL, EMBASE e MEDLINE, no ano de 2015. Foram analisados 20 estudos, dos quais 12 (60\%) relataram alta prevalência (75-100\%) do diagnóstico em distintos contextos clínicos, geralmente associado ao impedimento verbal por barreiras físicas, tais como: intubação/traqueostomia e alterações neurológicas. O diagnóstico Comunicação Verbal Prejudicada tem sido utilizado na prática clínica de maneira superficial, considerando principalmente aspectos físicos, de fácil identificação, sem avaliação mais precisa e detalhada do prejuízo na comunicação. Aspectos emocionais e sociais não foram considerados pela maioria dos estudos.

Descritores: Diagnóstico de Enfermagem; Comunicação; Enfermagem.

This is a study has as aim to identify in the literature the use in clinical practice of nursing diagnosis Impaired Verbal Communication in adults. It is an integrative literature review in the databases LILACS, BDENF, Coleciona SUS, CINAHL, EMBASE e MEDLINE, in 2015. 20 studies were analyzed, of which 12 (60\%) reported high prevalence $(75-100 \%)$ diagnosis in different clinical contexts, usually associated with the verbal impediment by physical barriers such as intubation/tracheostomy and neurological disorders. Diagnosis Impaired Verbal Communication has been used in clinical practice superficially, considering mainly physical aspects, easy identification without a more precise and detailed evaluation of damage in the communication. Emotional and social aspects were not considered in most studies.

Descriptors: Nursing Diagnosis; Communication; Nursing.

Este estudio tiene como objetivo identificar en la literatura el uso en la práctica clínica del diagnóstico de enfermería Comunicación Verbal Perjudicada en los adultos y personas mayores. Tratase de una revisión integradora de la literatura en las bases de datos LILACS, BDENF, Coleciona SUS, CINAHL, EMBASE e MEDLINE, en el año de 2015. Se analizaron 20 estudios, de los cuales 12 (60\%) informaron alta prevalencia (75-100\%) del diagnóstico en diferentes contextos clínicos, generalmente asociada al impedimento de la verbalización por barreras físicas como la intubación/traqueotomía y trastornos neurológicos. El diagnóstico Comunicación Verbal Perjudicada se ha utilizado en la práctica clínica de manera superficial, considerando principalmente aspectos físicos, de fácil identificación, sin evaluación más precisa y detallada del perjuicio en la comunicación. Aspectos emocionales y sociales no fueron considerados en la mayoría de los estudios.

Descriptores: Diagnóstico de Enfermería; Comunicación; Enfermería.

\footnotetext{
1Enfermeira. Mestre Enfermagem em Saúde do Adulto. Doutora e Pós Doutora em Enfermagem. Docente do Curso de Mestrado em Enfermagem da Universidade de Guarulhos. Professora Adjunta da Faculdade de Medicina de Jundiaí. Líder do Grupo de Pesquisa em Comunicação em Saúde. apuggina@prof.ung.br. Brasil.

${ }^{2}$ Enfermeira. Especialista em Cuidados Paliativos. Mestre em Enfermagem. Doutora em Ciências. Docente da Universidade de Guarulhos e Universidade São Judas Tadeu. Vice-líder do Grupo de Pesquisa em Comunicação em Saúde. Brasil.

${ }^{3}$ Enfermeira. Especialista em Terapia Intensiva Adulto. Especialista em Cuidados Paliativos. Coordenadora de Enfermagem do Instituto do Câncer do Hospital das Clínicas de São Paulo. Membro do Grupo de Pesquisa em Comunicação em Saúde. . Brasil.

4Enfermeira. Especialista em Enfermagem Clínica e Cirúrgica. Especialista em Transplantes de Órgãos. Especialista em Cuidados Paliativos. Mestre em Saúde do Adulto. Coordenadora de Enfermagem do Hospital Alemão Osvaldo Cruz. Membro do Grupo de Pesquisa em Comunicação em Saúde. Brasil.

5Enfermeira. Membro do Grupo de Pesquisa em Comunicação em Saúde. Brasil.

${ }^{6}$ Enfermeira. Mestre e Doutora em Enfermagem. Livre Docente e Professora Titular da Escola de Enfermagem da Universidade de São Paulo. juliaps@usp.br. Brasil.
} 


\section{INTRODUÇÃO}

$\mathrm{A}^{\mathrm{s}}$ Comunicação é a troca de mensagens que exercem influências no comportamento das pessoas envolvidas no processo e, é por meio da habilidade de comunicar-se que o homem se relaciona e transmite os seus conhecimentos para o mundo $^{1}$. Por tratar-se de um processo dinâmico que envolve um intercâmbio de mensagens enviadas e recebidas que influenciam no comportamento das pessoas a curto, médio e longo prazo é parte essencial no atendimento do cuidado humanizado ${ }^{2}$.

Esta, não apenas como instrumento básico para o relacionamento terapêutico, mas como competência ou capacidade interpessoal, permite ao profissional decodificar, decifrar e perceber mensagens emitidas pelo paciente e sua família, conduzindo-os para melhor interação e efetividade no cuidado prestado ${ }^{3}$. Está na base das habilidades do profissional de saúde, sendo relevante para os serviços de prevenção e para a prática diária. Usada não somente para o levantamento da história básica e demais dados, a comunicação é importante para a construção da relação com o paciente, na criação do vínculo, negociação e parceria 4 .

Não pode ser reduzida às generalizações interpretativas, visto tratar-se de um processo complexo, único na sensibilidade de quem comunica como também de quem se deixa afetar pela comunicação. Indagar, portanto, sobre a comunicação e seus múltiplos sentidos no ambiente hospitalar remete a problematizar a centralidade do papel do enfermeiro no cuidado e, consequentemente, situar a dimensão técnica que envolve os processos e diagnósticos de enfermagem neste processo de cuidar.

A internação hospitalar é percebida como sendo uma experiência desagradável por quem a vivencia, uma vez que é permeada pelo medo do desconhecido, pela utilização de recursos tecnológicos muitas vezes invasivos e dolorosos, pelo uso de linguagem técnica e rebuscada que aumenta a ansiedade do ser doente no que tange a seu quadro patológico, pela inquietação em estar em um ambiente estranho de estruturas rígidas que o descaracteriza, partilhando o mesmo espaço físico com pessoas fora de seu convívio familiar e, ainda, pela preocupação com sua evolução clínica ${ }^{5}$.

Para o ser humano, a doença é a quebra da harmonia orgânica, interferindo, com todos os setores da sua vida, especialmente na convivência com os familiares mais próximos. Diante dessas possibilidades, a equipe de enfermagem procura amenizar as sensações de desequilíbrio bio-psico-sócio-espirituais apresentadas pelo paciente, aumentando sua confiança e a autoestima. A relação interpessoal que se dá entre o enfermeiro e a pessoa hospitalizada está calçada na comunicação entre ambos ${ }^{6}$.

A comunicação é um instrumento básico para o cuidado humanizado; ela deve estar presente em todas as ações realizadas com o paciente, seja para orientar, informar, apoiar ou confortar. É por meio da comunicação que as pessoas podem expressar o que são, relacionar-se, satisfazer suas necessidades. Por isso, o relacionamento entre enfermeiro e paciente adquire tanta importância no fenômeno de cuidar? .

Quando se estabelece o processo de comunicação com o outro é importante pensar que o ato de se comunicar é amplo, ele não se inicia e ou se encerra na palavra, que não é tudo na comunicação. Quando duas ou mais pessoas conversam, ambos processam as palavras e as mensagens e constroem seus próprios sentidos para aquilo que foi conversado, (re) construindo conhecimentos e pensamentos sobre outras coisas, sucessivamente. Logo, a comunicação imprime marcas nos sujeitos. Tanto a comunicação verbal como a não verbal, pois a linguagem do corpo se expressa nos gestos, nas expressões e nas emoções ${ }^{8}$.

Existe apenas um diagnóstico de enfermagem na NANDA (North American Nursing Diagnosis Association) relacionado a problemas no processo de comunicação, qual 
seja, o diagnóstico Comunicação Verbal Prejudicada. Esse diagnóstico tenta abordar a questão da comunicação como um todo. 0 diagnóstico Comunicação Verbal Prejudicada foi incluído na classificação em 1983 e revisto em 1996 e novamente em 1998. É definido por habilidade diminuída, atrasada ou ausente para receber, processar, transmitir e usar um sistema de símbolos ${ }^{9}$.

A adoção do diagnóstico de enfermagem Comunicação Verbal Prejudicada como instrumento tecnológico para o cuidado ao paciente impossibilitado de verbalizar pode orientar a prática profissional crítica, reflexiva e individualizada do enfermeiro. Permite aprimorar, direcionar e qualificar suas ações assistenciais em prol da melhora na expressão e interação. 0 uso de nomenclatura única para descrever o prejuízo da comunicação do paciente possibilita ainda ao enfermeiro, padronizar seus registros quanto às necessidades de cuidado, permitindo avaliação futura da adequação de medidas que visem suprir as necessidades de cuidado identificadas.

Um paciente com o diagnóstico de Enfermagem Comunicação Verbal Prejudicada precisa de uma assistência específica, efetiva e que proporcione bem-estar, visto que comunicação é uma necessidade humana inerente e extremamente importante no processo de restabelecimento da saúde.

O objetivo deste estudo foi identificar na literatura o uso na prática clínica do diagnóstico de enfermagem Comunicação Verbal Prejudicada em adultos e idosos.

\section{MÉTODO}

Trata-se de um estudo de revisão integrativa da literatura, método que consiste na análise de textos com o propósito de reunir e sintetizar resultados de estudos anteriores sobre determinado assunto, com vistas a obter profundo conhecimento do tema investigado, além de determinar se o conhecimento é válido para ser aplicado à prática ${ }^{10}$.

Para a construção desse tipo de revisão é necessário seguir um processo de seis etapas, que foram adotadas neste estudo, a saber: identificação do tema e pergunta de pesquisa; definição dos critérios de inclusão e exclusão dos estudos e amostra; síntese dos estudos selecionados em formato de tabelas, concebendo um banco de dados; análise crítica dos achados, identificando diferenças e conflitos; interpretação dos resultados; e, a apresentação da revisão ${ }^{10}$.

No mês de novembro de 2015 foi realizada busca integrada com o recurso boleano $A N D$ e o uso das palavras-chave comunicação, verbal e prejudicada no portal BVS (Biblioteca Virtual da Saúde), considerando as bases de dados LILACS (Literatura Latino-Americana e do Caribe em Ciências da Saúde), BDENF e Coleciona SUS (Brasil). Nas bases de dados da CINAHL, EMBASE e no portal da PubMed, considerando a base de dados MEDLINE, a busca integrada utilizou-se, além das palavras-chave communication, verbal e impaired, o descritor Nursing Diagnosis devido ao elevado número de artigos encontrado sem este descritor. As buscas não tiveram limitação de período.

Para a seleção dos textos foram considerados os seguintes critérios de inclusão: estudo na íntegra em português, espanhol ou inglês; em que fosse apresentado uso ou aplicação do diagnóstico de enfermagem Comunicação Verbal Prejudicada, considerando a população de adultos e idosos.

Após leitura informativa dos artigos foi possível a captura na íntegra daqueles que atendiam aos critérios de inclusão. Com 0 intuito de organizar os dados foi elaborado um instrumento (quadro resumo), que destacava os dados de identificação do artigo (título, autores, periódico, ano de publicação e idioma), objetivo, método, principais resultados. Para a análise dos dados, foram realizadas repetidas leituras exploratórias e críticas de todo o conteúdo.

\section{RESULTADOS}

No portal BVS (Biblioteca Virtual da Saúde), obteve-se o resultado inicial de 38 estudos, sendo 21 na base de dados LILACS, 16 na 
BDENF e 1 publicação na base Coleciona SUS (Brasil). Nas bases de dados da CINAHL, EMBASE e MEDLINE os resultados foram respectivamente 13, 1 e 9 artigos. 0 total desta etapa de busca foram 61 publicações.

Nas etapas seguintes, 26 artigos foram excluídos por motivo de repetição, permanecendo 35 para a análise posterior dos títulos e resumos. Nas etapas de leitura pelos resumos e texto na íntegra foram excluídos respectivamente 9 e 6 estudos (Tabela 1). Portanto, 16 estudos foram selecionados para análise, preenchimento do instrumento de organização de dados e inclusão (Figura 1 e Quadro 1).

Figura 1. Sequência ilustrativa das etapas da revisão integrativa. São Paulo, 2015.

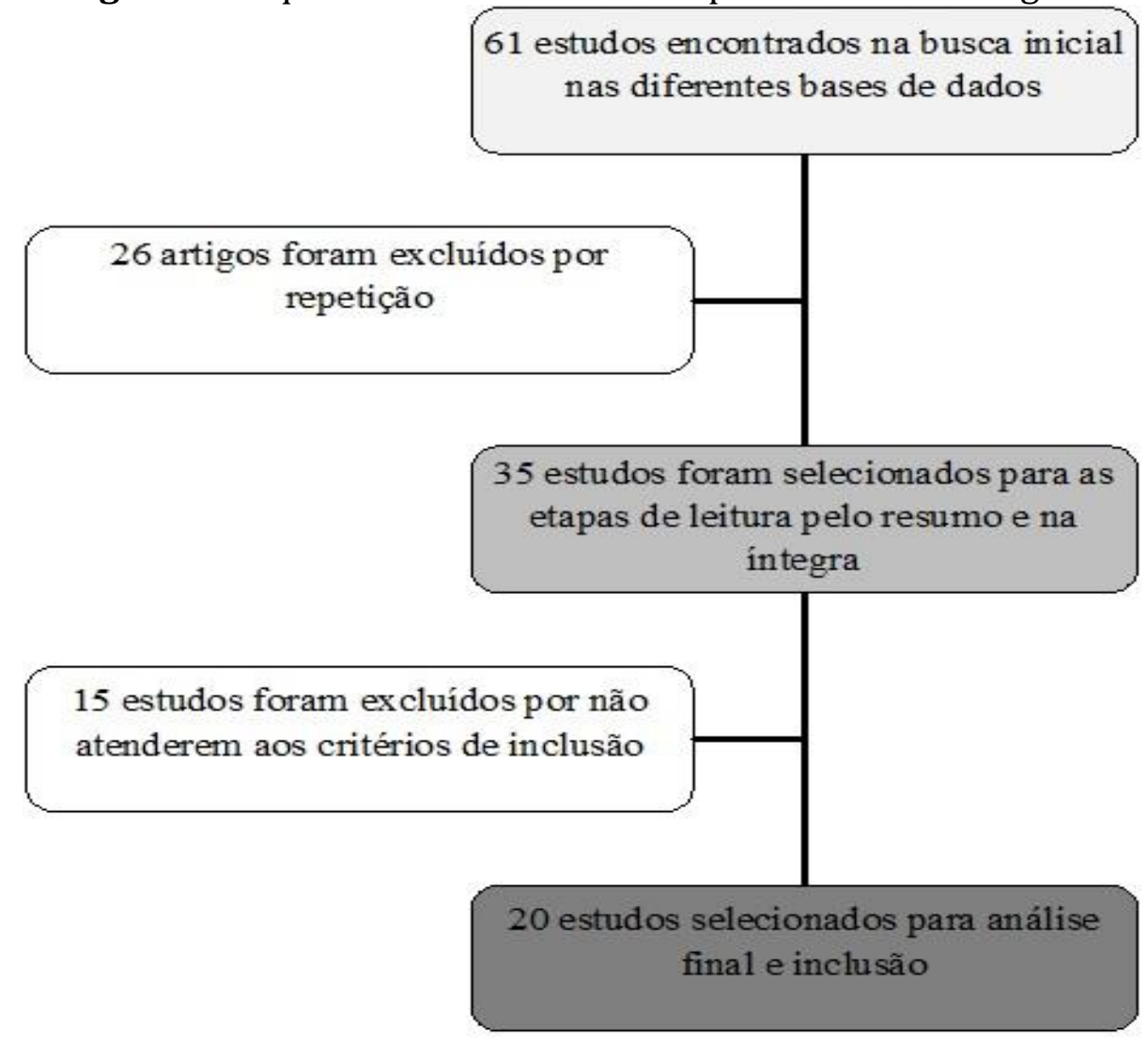

Tabela 1. Motivo de exclusão dos artigos. São Paulo, 2015.

\begin{tabular}{ll}
\hline Motivo da exclusão \\
\hline Idioma* $^{*}$ \\
População** \\
Não aplica o diagnóstico \\
Publicação de resumo em \\
Anais \\
*OTAL \\
\hline Chinês, Turco e Dutch (n=3); **Crianças (n=2).
\end{tabular}


Quadro 1. Estudos selecionados para a revisão integrativa. São Paulo, 2015.

\section{TíTULO DOS ESTUDOS}

- Diagnósticos e intervenções de enfermagem frequentes em mulheres internadas em uma unidade de terapia intensiva ${ }^{11}$.

- Comunicação verbal prejudicada da família: evidenciando a necessidade de desenvolver um novo diagnóstico de enfermagem ${ }^{12}$.

- Comunicação verbal prejudicada: atividades empregadas vs. atividades propostas na literatura ${ }^{13}$.

- Assistência de enfermagem no pós-operatório de transplante hepático: identificando diagnósticos de enfermagem ${ }^{14}$.

- Diagnósticos de enfermagem em idosos dependentes residentes em uma instituição de longa permanência em Fortaleza-CE 15 .

- Diagnósticos e intervenções de enfermagem em pacientes submetidos a laringectomia total e parcial ${ }^{16}$.

- Enfermagem em laboratório de hemodinâmica: diagnóstico e intervenção fundamentados na

Teoria da Adaptação de Roy ${ }^{17}$.

- 0 enfermeiro e o diagnóstico de enfermagem: comunicação verbal prejudicada ${ }^{18}$.

- Ventilatory weaning: a case study of protracted weaning ${ }^{19}$.

- Aplicação do Processo de Enfermagem: estudo de caso com uma puérpera ${ }^{20}$.

- Avaliação de famílias no contexto hospitalar: uma aproximação entre o modelo Calgary e a taxonomia da NANDA 21.

- Diagnósticos de enfermagem em pacientes com acidente vascular cerebral ${ }^{22}$.

- Principais diagnósticos de enfermagem em pacientes portadores da síndrome da imunodeficiência adquirida (AIDS) ${ }^{23}$.

- A comunicação no relacionamento interpessoal enfermeiro/cliente idoso oncológico submetido a traqueostomia de emergência ${ }^{24}$.

- Diagnósticos de enfermagem em clientes internadas em unidade médico-cirúrgica ${ }^{25}$.

- Impaired verbal communication during short-term oral intubation ${ }^{26}$.

- Comunicação verbal prejudicada: investigação no período pós-acidente vascular encefálico ${ }^{27}$.

- Assistência de enfermagem a clientes com câncer na cabeça e no pescoço com ênfase nos tumores de cavidade oral no Estado do Rio de Janeiro ${ }^{28}$.

- Plan de cuidados de un paciente con encefalopatía anóxica ${ }^{29}$.

- Nursing diagnosis in intensive care unit: The Turkey experience ${ }^{30}$.

Todos os estudos analisados tinham enfermeiros como autores, 18 (90\%) foram publicados em periódicos de Enfermagem ${ }^{11-}$ 15,17-23,25-30, assim como 16 (80\%) foram desenvolvidos no Brasil e publicados na língua portuguesa ${ }^{11-18,20-28 .}$

Por meio de leitura crítica e analítica dos estudos foi possível identificar que, embora todos versem sobre o prejuízo da comunicação verbal em distintas situações da prática clínica, 12 (60\%) exploram o diagnóstico de Enfermagem Comunicação Verbal Prejudicada de modo secundário e superficial, por meio da identificação de sua prevalência ou incidência em distintas populações de pacientes ${ }^{11,14-18,21-23,25,28,30}$.
Três trabalhos (15\%) são descritos como estudos de caso, nos quais há relato da presença do referido diagnóstico inferido por meio da caracterização da condição clínica dos pacientes estudados ${ }^{19,20,29}$; outros dois $(12,5 \%)$ são pesquisas qualitativas, que citam o contexto e a vivência de condições que impedem a verbalização por meio da ótica do enfermeiro ${ }^{24}$ e de pacientes ${ }^{26}$. Apenas três (15\%) estudos têm como foco principal a exploração aprofundada na prática clínica do diagnóstico de Enfermagem Comunicação Verbal Prejudicada ${ }^{12,13,27}$.

Os estudos analisados apontam alta prevalência do diagnóstico Comunicação Verbal Prejudicada. 0 mesmo mostrou-se prevalente em 28 (100\%) mulheres com tempo de internação maior do que três dias em 
UTI obstétrica em hospital do Ceará11; em 14 (100\%) pacientes em pós operatório imediato de transplante hepático, também em instituição hospitalar no referido Estado ${ }^{14}$; em $10(100 \%)$ doentes internados em UTI neurológica por acidente vascular encefálico, em Santos (SP) ${ }^{22} ; 25 \quad(86,2 \%)$ idosos dependentes e com condições crônicas de doença em Instituição de Longa Permanência ${ }^{15} ; \quad 48(80 \%)$ pacientes com HIV/AIDS internados em unidade hospitalar de doenças infecciosas, em São Paulo23; 184 $(78,5 \%)$ pessoas com doença coronariana aguda atendidas no serviço de hemodinâmica, no Ceará17. E, prevalência deste diagnóstico em Goiás em 12 (75\%) unidades familiares de pacientes atendidos em unidade de internação de Hospital Escola ${ }^{23}$.

Quanto à incidência, pesquisa realizada em unidades de cuidados críticos e de internação de instituição hospitalar de MaríliaSP evidenciou que, durante 852 dias, apenas a média de 0,015 pacientes tiveram atribuído o diagnóstico Comunicação Verbal Prejudicada, a despeito da média de 10,9 pacientes submetidos à intubação orotraqueal (IOT)/dia. No referido estudo, os autores atribuem pouca consideração à capacidade de comunicação verbal do paciente pelo enfermeiro na prática clínica, sugerindo maior destaque às questões de cunho biologico ${ }^{18}$.

Nos três estudos de caso analisados, o diagnóstico Comunicação Verbal Prejudicada é descrito em contextos semelhantes, de atenção a pacientes críticos com doença em estado avançado. 0 primeiro é um estudo britânico ${ }^{19}$ que descreve as condições e evolução clínica de uma paciente com câncer de pulmão e resposta disfuncional ao desmame ventilatório, inicialmente submetida à IOT e, posteriormente, à traqueostomia. As autoras destacam que este diagnóstico é comum no referido contexto de dificuldade de desmame ventilatório, uma vez que, por terem o nível de sedação gradativamente diminuído, tornam-se alertas, porém impedidos de verbalizar, podendo causar frustração e ansiedade no indivíduo ${ }^{19}$.
Outro estudo de caso $^{20}$, realizado no Brasil, traz o diagnóstico Comunicação Verbal Prejudicada como coadjuvante na descrição da implementação do Processo de Enfermagem à puérpera internada em UTI devido miocardiopatia pós-parto e encefalopatia hipóxica severa pós parada cardiorespiratória. As autoras associam a presença do diagnóstico às alterações neurológicas da paciente, em estado de coma vigil, e listam intervenções de enfermagem e resultados esperados relacionados à comunicação na abordagem da paciente, baseadas nas nomenclaturas de enfermagem ${ }^{20}$.

0 terceiro estudo de caso analisado ${ }^{30}$ envolve simulação de caso clínico e enfoca a descrição de diagnósticos de enfermagem atribuídos por enfermeiras intensivistas da Turquia à paciente hipotético. Os autores relatam que os diagnósticos apontados com maior frequência foram relacionados à integridade da pele e da mucosa oral, sendo o diagnóstico Comunicação Verbal Prejudicada o que obteve menor índice de identificação, apenas 2,2\% $(n=45)$. Destaque relevante do trabalho refere-se ao fato de que nenhum diagnóstico de cunho psicossocial foi apontado pelos enfermeiros, denotando, segundo os pesquisadores, pouca valorização aos aspectos culturais e sociais e às dimensões emocional e espiritual do paciente.

Um dos estudos qualitativos analisados é brasileiro e foi desenvolvido por meio de entrevistas com 15 enfermeiras de hospital oncológico, envolvidas no cuidado de pacientes idosos sob IOT. Embora traga no título o termo comunicação com pacientes traqueostomizados, oferece pouco destaque ao diagnóstico Comunicação Verbal Prejudicada neste contexto específico. Apenas cita na discussão de uma das categorias temáticas, denominada comunicação verbal, que o referido diagnóstico é uma ferramenta que pode auxiliar o enfermeiro a implementar intervenções de enfermagem, sem exploração mais aprofundada acerca de sua aplicação ${ }^{24}$.

0 outro estudo qualitativo ${ }^{26}$ foi desenvolvido em Nova Jersey, EUA, por meio 
de entrevistas com dez pacientes que tinham sido submetidos à IOT. Estes descreveram a experiência de estar impossibilitados de verbalizar como assustadora e frustrante, revelando que o que mais gostariam de comunicar aos profissionais nesta situação era a dor e o desconforto. Apesar de ter sido publicado em periódico especializado em diagnósticos de enfermagem e trazer no título da publicação a terminologia, utilizando a nomenclatura da NANDA, o estudo não aborda objetivamente a utilização do diagnóstico, destacando apenas o prejuízo da comunicação verbal nos pacientes intubados.

0 diagnóstico de enfermagem Comunicação Verbal Prejudicada foi abordado com aprofundamento em pesquisa descritiva realizada com a unidade familiar de pacientes internados em hospital de Goiás (GO). Os discursos dos familiares foram registrados e analisados segundo agrupamento por familiaridade, sob os mesmos foi aplicado raciocínio diagnóstico e a taxonomia, o que possibilitou a inferência e proposta dos autores de um novo diagnóstico: comunicação verbal prejudicada da família. Foi definido com base na adaptação do conceito da NANDA, do indivíduo para a unidade familiar e proposto tendo como fatores relacionados aqueles encontrados com maior frequência nas famílias estudadas: barreiras psicológicas envolvendo histórias de conflitos, a falta de vínculos e de modelos familiares. 0 estudo traz recomendações de adaptações e refinamentos ao diagnóstico Comunicação Verbal Prejudicada, com vistas ao atendimento da família ${ }^{12}$.

Outra pesquisa com abordagem central do referido diagnóstico foi realizada em Ribeirão Preto - SP, em três etapas. A primeira, envolvendo revisão de literatura, evidenciou a relação entre o diagnóstico e suas intervenções específicas, sendo a maioria destas descritas segundo a Classificação de Intervenções de Enfermagem (NIC). Na segunda fase houve mapeamento das intervenções propostas e validação por comitê de juízes de novas atividades relacionadas especificamente ao prejuízo da comunicação verbal. Na terceira etapa, enfermeiros foram questionados sobre o uso do diagnóstico e intervenções relacionados à comunicação verbal prejudicada e $69 \%$ referiu utilizá-los, sendo a ação mais empregada a escuta ativa $(76,6 \%)$. Os autores propõem inclusão de outras atividades associadas às intervenções para o diagnóstico Comunicação Verbal Prejudicada.

0 enfoque aprofundado ao prejuízo da comunicação verbal foi identificado também em estudo realizado em Fortaleza (CE) ${ }^{27}$. Embora os autores descrevessem que o objetivo da pesquisa era investigar a prevalência do diagnóstico Comunicação Verbal Prejudicada em pacientes em processo de reabilitação devido acidente vascular encefálico, também apontaram, de modo detalhado, as características definidoras que foram utilizadas para embasar o processo de raciocínio clínico para atribuição do referido diagnóstico, correlacionando-as com distintas variáveis.

Assim, destacaram além da prevalência de $37,5 \% \quad(n=40)$, associações estatísticas significantes entre a orientação tempo/espaço/pessoa com a dificuldade para expressar verbalmente os pensamentos $(\mathrm{p}=0,006)$ e com a pronúncia indistinta $(p=0,007)$. Outro dado relevante apontado por este estudo foi que as características definidoras dificuldade de usar a expressão corporal e dificuldade de usar a expressão facial foram as terceiras mais frequentemente encontradas, ambas presentes em $73,3 \%$ dos pacientes com prejuízo da comunicação verbal estudados $(n=15)$ e que, contudo, referem-se à dimensão não verbal do processo comunicativo $^{27}$.

Com relação às características definidoras e fatores relacionados ao diagnóstico Comunicação Verbal Prejudicada há poucas referências dos mesmos nos estudos analisados, exceto no último descrito ${ }^{30}$. Como grande parte se refere ao contexto de pacientes com verbalização prejudicada devido intubação ou traqueostomia, há indícios que os fatores relacionados mais frequentes sejam 
aqueles atrelados à presença de barreiras físicas $11,14,18,19,22,26$ e condições fisiológicas /neurológicas alteradas $11,15,17,19,20,23,27-29$.
A Figura 2 ilustra os principais resultados da referida revisão.

Figura 2. Esquema ilustrativo dos principais resultados da revisão de literatura. São Paulo, 2015.

\section{Diversidade de fatores relacionados vinculados ao diagnóstico} de enfermagem Comunicação Verbal Prejudicada

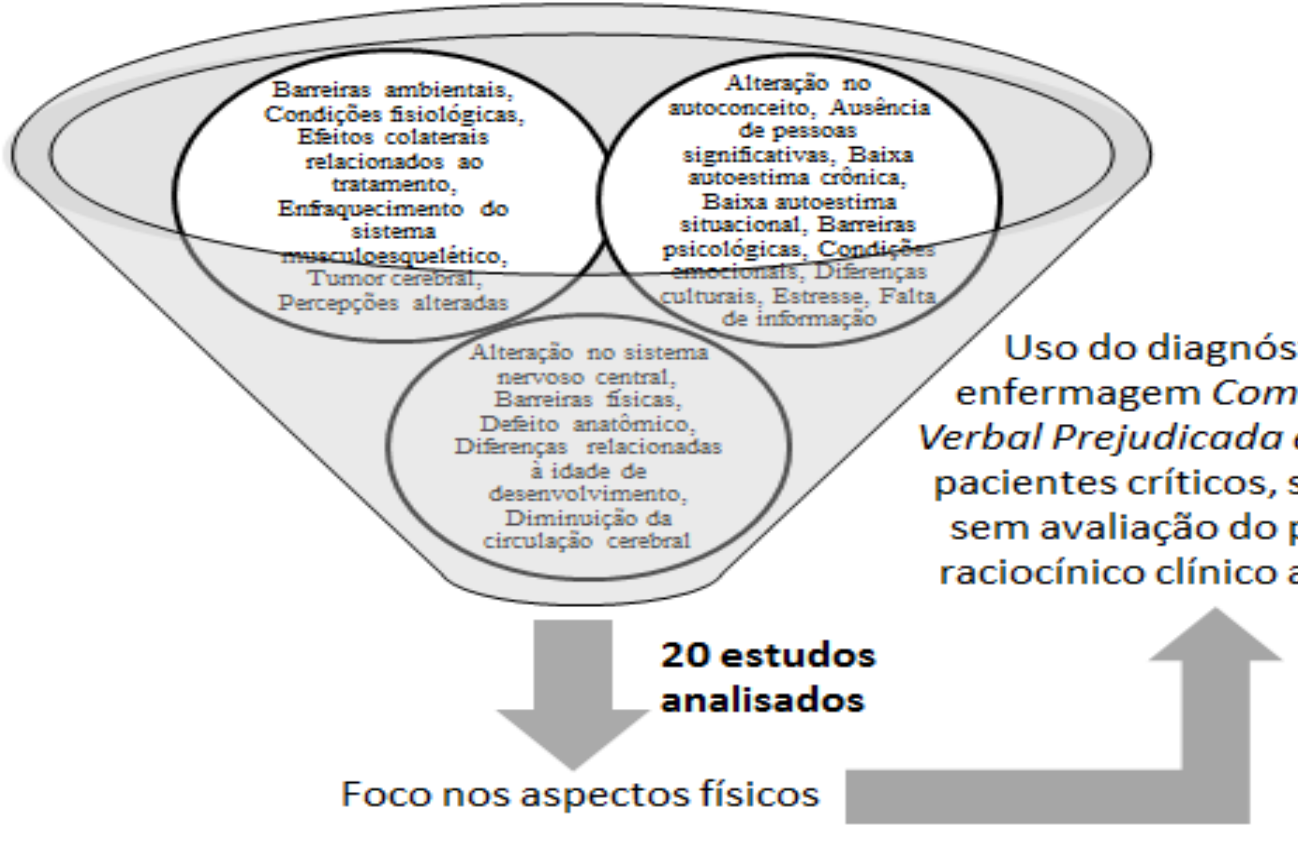

\section{DISCUSSÃo}

Os autores não exploram com aprofundamento os prejuízos verbais, as discussões são superficiais e o foco assistencial é sobre aspectos físicos e facilmente identificados, sem a implementação de avaliação e de raciocínio clínicos adequados. Por isso este estudo, apesar de suas limitações relacionadas ao acesso digital e idioma, pode trazer contribuições à ciência da enfermagem, uma vez que aponta alta prevalência do diagnóstico em distintos contextos e situações clínicas em que é possível sua utilização. Contudo, outras pesquisas são necessárias, considerando a complexidade do processo comunicacional, sem redução apenas à dimensão verbal.

No exercício do cuidado é fundamental para o enfermeiro conhecer e aplicar em sua prática clínica a nomenclatura diagnóstica de enfermagem adequada a situações em que há prejuízo da comunicação verbal.

Contudo, os estudos analisados nesta revisão trazem, em sua maioria, dados de prevalência, sem explorar com o merecido aprofundamento o contexto de aplicação do diagnóstico, com suas características definidoras e fatores relacionados, apontando as potencialidades e fragilidades do mesmo. E, nota-se que os estudos exploram o prejuízo da comunicação no contexto do cuidado crítico ou de atenção a indivíduos com agravos físicos/fisiológicos, denotando carência de exploração desta condição na saúde mental e psiquiátrica, áreas nas quais a prática clínica mostra a necessidade de identificação de problemas relativos à comunicação. Também não foram encontrados estudos que tenham realizado ou, ao menos, sugerido a validação de conteúdo do diagnóstico Comunicação Verbal Prejudicada.

Por ser o único diagnóstico que abarca a dimensão comunicativa do ser humano na atual classificação taxonômica da NANDA, o diagnóstico Comunicação Verbal Prejudicada parece estar sendo pouco explorado na literatura. E, justamente por ser ímpar na 
complexa dimensão comunicativa, parece necessária a consideração da dimensão não verbal com maior propriedade, tal sua relevância.

Para que as informações possam ser devidamente compartilhadas, o enfermeiro deve lembrar-se que a comunicação se processa além das palavras, representada pela linguagem verbal, e que o não verbal envolve: gestos, silêncios, expressões faciais, movimentos do corpo e distâncias mantidas entre as pessoas. 0 profissional deve ouvir atentamente, sabendo o que falar e quando falar, em linguagem clara e acessível, pois os pacientes e familiares, muitas vezes, por vergonha, medo, ansiedade, entre outros sentimentos, não expressam verbalmente suas necessidades ${ }^{31-34}$.

Assim, quando a comunicação verbal se encontra prejudicada, perceber e compreender o não verbal pode auxiliar o enfermeiro a identificar com maior precisão os sentimentos do paciente, suas dúvidas e dificuldades de verbalização, contribuindo para a prática da excelência da enfermagem ${ }^{35}$. Ajuda o profissional, ainda, a potencialização de sua própria comunicação, enquanto elemento transmissor de mensagens ${ }^{34}$. Deve-se ter por objetivo ajudar o cliente, de forma estruturada, por meio de interações planejadas, utilizandose os conhecimentos da comunicação terapêutica ${ }^{1}$.

\section{CONCLUSÃO}

O diagnóstico Comunicação Verbal Prejudicada tem sido utilizado na prática clínica de maneira superficial, considerando aspectos principalmente físicos, de fácil identificação, sem avaliação mais precisa e detalhada do prejuízo na comunicação. Aspectos emocionais e sociais não foram considerados pela maioria dos estudos.

\section{REFERÊNCIAS}

1. Stefanelli MC. Comunicação com o paciente: teoria e ensino. 2ed. São Paulo: Robe Editorial; 1993.

2. Machado ACA. Comunicação não verbal de idosos frente ao processo de dor. Rev Bras Enferm. 2006; 59(2):129-33.
3. Araújo MMT, Silva MJP, Puggina AC. A comunicação não verbal enquanto fator iatrogênico. Rev Esc Enferm USP. 2007; 41(3):419-25.

4. Rossi PS, Batista NA. O Ensino da Comunicação na graduação em medicina: uma abordagem. Interface Comum Saúde Educ. 2006; 10(19):93-102.

5. Morais GSN, Costa SFG, Fontes WD, Carneiro AD. Comunicação como instrumento básico no cuidar humanizado em enfermagem ao paciente hospitalizado. Acta Paul Enferm. 2009; 22(3):323-7.

6. Paula ADD, Furegato ARF, Scatena MCM. Interação enfermeiro-familiar de paciente com comunicação prejudicada. Rev. latinoam Enferm. 2000; 8(4):45-51.

7. Pontes IAC, Leitão IMTA, Ramos IC. Comunicação terapêutica em enfermagem: instrumento essencial do cuidado. Rev Bras Enferm. 2007; 61(3):312-8.

8. Ferreira MA. A comunicação no cuidado: uma questão fundamental na enfermagem. Rev Bras Enferm. 2006; 59(3):327-30.

9. Nanda International. Diagnósticos de enfermagem da NANDA: definições e classificação, 2012-2014. Porto Alegre: Artmed; 2013.

10. Mendes KDS, Silveira RCCP, Galvão CM. Revisão integrativa: método de pesquisa para a incorporação de evidências na saúde e na enfermagem. Rev Texto \& Contexto Enferm. 2008; 17(4):758-64.

11. Oliveira MF, Freitas MC. Diagnósticos e intervenções de enfermagem frequentes em mulheres internadas em uma unidade de terapia intensiva. Rev Bras Enferm. 2009; 62(3):343-8.

12. Montefusco SRA, Bachion MM, Carvalho EC, Munari DB. Comunicação verbal prejudicada da família: evidenciando a necessidade de desenvolver um novo diagnóstico de enfermagem. Ciênc Cuid Saúde. 2009; 8(4): 622-9.

13. Favretto DO, Carvalho EC, Canini SRMS, Garbin LM. Comunicação verbal prejudicada: atividades empregadas vs. atividades propostas na literatura. REME Rev Min Enferm. 2007; 11(3):227-32.

14. Ramos IS, Oliveira MAL, Braga VAB. Assistência de enfermagem no pós operatório de transplante hepático: identificando diagnósticos de enfermagem. Ciênc Cuid Saúde. 2011; 10(1):116-26.

15. Freitas MC, Pereira RF, Guedes MVC. Diagnósticos de enfermagem em idosos dependentes residentes em uma instituição de longa permanência em Fortaleza - CE. Ciênc Cuid Saúde. 2010; 9(3):518-26.

16. Carmagnani MIS. Diagnósticos e intervenções de enfermagem em pacientes submetidos à laringectomia total e parcial. [tese]. São Paulo: Universidade Federal de São Paulo, Escola Paulista de Medicina; 1999.

17. Oliveira MF, Silva LF. Enfermagem em laboratório de hemodinâmica: diagnóstico e intervenção 
fundamentados na Teoria da Adaptação de Roy. Rev Eletrônica Enferm. 2010; 12(4):678-85.

18. Marvulo MML, Barbosa PMK, Marvulo PL. O enfermeiro e o diagnóstico de enfermagem: comunicação verbal prejudicada. Nursing. 2009; 12(134):321-5.

19. Pattison N, Watson J. ventilatory weaning: a case study of protacted weaning. Nurs Crit Care. 2009; 14(2):75-85.

20. Alves AR, Chaves EMC, Freitas MC, Monteiro ARM. Aplicação do processo de Enfermagem: estudo de caso com uma puérpera. Rev Bras Enferm. 2007; 60(3):344-7.

21. Montefusco SRA, Bachion MM, Nakatani AYK. Avaliação de famílias no contexto hospitalar: uma aproximação entre o modelo Calgary e a taxonomia da NANDA. Texto \& Contexto Enferm. 2008; 17(1):7280.

22. Mattia AL, Barbosa MH, Pontes DC, Alves DC. Diagnósticos de enfermagem em pacientes com acidente vascular cerebral. Nursing. 2011; 14(158):388-94.

23. Silva MR, Bettercourt AR, Diccini S, Belasco A, Barbosa DA. Diagnósticos de enfermagem em portadores da síndrome da imunodeficiência adquirida. Rev Bras Enferm. 2009; 62(1):92-9.

24. Alves DY. A comunicação no relacionamento interpessoal enfermeiro /cliente idoso oncológico submetido à traqueostomia de urgência. [Dissertação]. Rio de Janeiro (RJ): Universidade Federal do Estado do Rio de Janeiro; 2008.

25. Volpato MP, Cruz DALM. Diagnósticos de enfermagem de pacientes internadas em unidade médico cirúrgica. Acta Paul Enferm. 2007; 20(2):11924.

26. Fowler SB. Impaired verbal communication during short-term oral intubation. Nurs Diagn. 1997; 8(3):93-8.

27. Chaves DBR, Costa AGS, Oliveira ARS, Silva VM, Araujo TL, Lopes MVO. Comunicação verbal prejudicada: investigação no período pós-acidente vascular encefálico. Rev RENE. 2013; 14(5):877-85.
28. Raimundo DD, Guedes MTS, Luzial NS, Peixoto MGS, Santos MCM, Silva CC. Assistência de enfermagem a clientes com câncer na cabeça e no pescoço com ênfase nos tumores de cavidade oral no Estado do Rio de Janeiro. Rev Pesqui Cuid Fundam. 2014; 6(4):1496-504.

29. Lendinez Mesa A, Casero Alcazar M, Fernandes Ribeiro AS. Care plan for a patient with anoxic encephalopathy. Rev Cient Soc Esp Enferm Neurol. 2014; 39(1):29-33.

30. Korhan EA, Yönt GH, Erdemir F, Müller-Staub M. Nursing diagnosis in intensive care unit: The Turkey experience. Crit Care Nurs Q. 2014; 37(2):219-24.

31. Bezerra ALQ, Dal Ben LW, Camargo MNV, Pinheiro VFO. Gestos e posturas do enfermeiro durante a orientação a familiares de pacientes internados em unidade de terapia intensiva (UTI). Rev Esc Enferm USP. 1998; 32(2):134-9.

32. Leal MII et al. Percepções da equipe de enfermagem de unidade de terapia intensiva. Nursing. 2006; 94(9):719-23.

33. Durman S, Stefanelli MC. Enfermeiros de hospital geral construindo conceitos para o desenvolvimento da relação de ajuda terapêutica. Texto \& Contexto Enferm. 2000; 9 (2 Pt 2):714-25.

34. Silva MJP. Comunicação tem remédio: a comunicação nas relações interpessoais em saúde. 8ed. São Paulo: Loyola; 2012.

35. Inaba LC, Silva MJP, Telles SCR. Paciente crítico e comunicação: visão de familiares sobre sua adequação pela equipe de enfermagem. Rev Esc Enferm USP. 2005; 39(4):423-9.

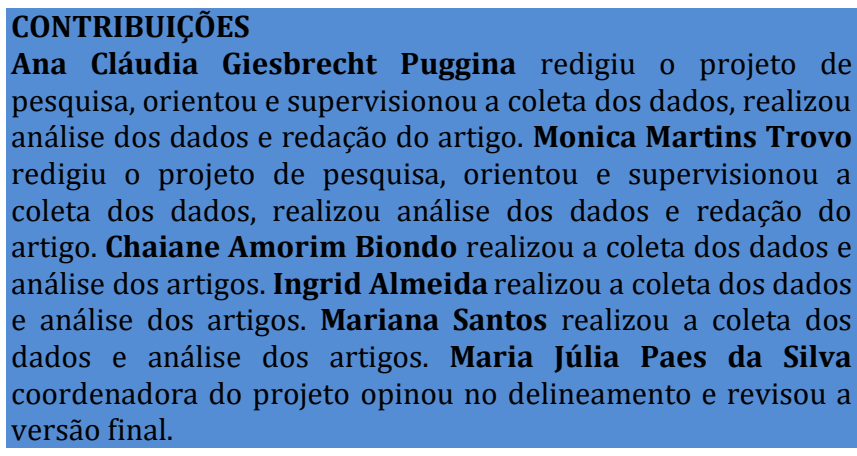

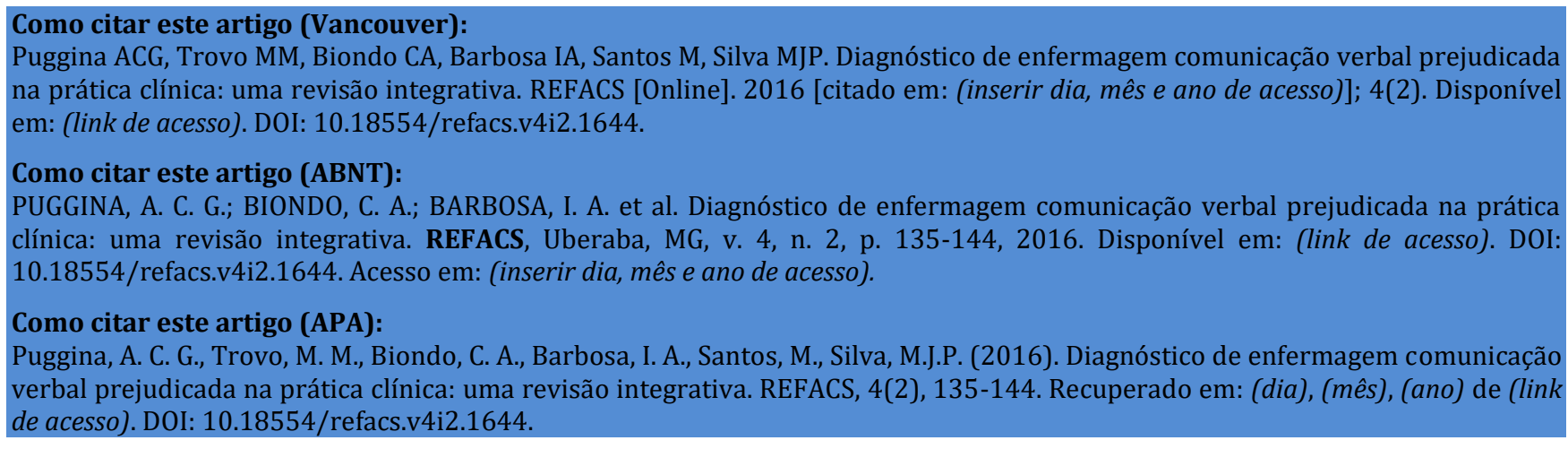

